\title{
Prolonged Pyrexia due to Hemophaghocytic Lymphohistiocytosis
}

Farzana Shumy ${ }^{1}$, Tanvir Ahmed ${ }^{2}$, Ahmad Mursel Anam³, Abed Hussain Khan ${ }^{4}$, Mohammad Ferdous Ur Rahaman $^{5}$, Quazi Mamtaz Uddin Ahmed ${ }^{6}$, M A Jalil Chowdhury ${ }^{7}$

\begin{abstract}
:
Haemophaghocytic lymphohistiocytosis (HLH) is an uncommon illness in adults. It is rare but common clinical features like lymphadenopathy, hepatosplenomegaly, fever are presentation of many different familiar clinical illness, make it a diagnostic challenge. Here, we present a case of a previously healthy young boy presented with fever and other features like hepatosplenomegaly, pancytopenia, markedly elevated serum ferritin, Lactate dehydrogenase, triglyceride, low fibrinogen, persistent hyponatremia and finally detectable Haemophagocytosis in his bone marrow. Unfortunately, diagnosis was delayed around 4 month after his initial presentation and he succumbed to his disease. This case highlights common clinical features and diagnostic difficulties have to face in making confirmed diagnosis of HLH.
\end{abstract}

Key Words: Haemophaghocytic lymphohistiocytosis, Fever, Pancytopenia

\section{Introduction:}

Haemophaghocytic lymphohistiocytosis (HLH) is a rare disorder characterized by prolonged fever, cytopenias, hepatosplenomegaly, hypertriglyceridemia, disseminated with a variety of disorder like viral, bacterial, fungal and liver or lymphatic nodes histiocytosis. It has been associated with coagulopathy and bone marrow, spleen, parasitic infections

1 Dr. Farzana Shumy, MBBS, FCPS (Medicine), Medical Officer, Department of Internal Medicine, Bangabandhu Sheikh Mujib Medical University, Shahbagh, Dhaka 1000, Bangladesh.

2 Dr. Tanvir Ahmed, MBBS, Resident, Department of Oncology, Bangabandhu Sheikh Mujib Medical University, Shahbagh, Dhaka 1000 , Bangladesh

3 Dr. Ahmad Mursel Anam, MBBS, Chief Resident, ICU, Square Hospitals Ltd. 18/F, BU Qazi Nuruzzaman Sarak (West Panthapath), Dhaka 1205, Bangladesh.

4 Dr. Abed Hussain Khan, MBBS, FCPS (Medicine), Medical Officer, Department of Internal Medicine, Bangabandhu Sheikh Mujib Medical University, Shahbagh, Dhaka 1000, Bangladesh.

5 Dr. Mohammad Ferdous Ur Rahaman, MBBS, FCPS (Medicine), Assistant Professor, Department of Internal Medicine, Bangabandhu Sheikh Mujib Medical University, Shahbagh, Dhaka 1000 , Bangladesh.

6 Dr. Quazi Mamtaz Uddin Ahmed, MBBS, FCPS (Medicine), Associate Professor, Department of Internal Medicine, Bangabandhu Sheikh Mujib Medical University, Shahbagh, Dhaka 1000, Bangladesh.

7 Prof. M A Jalil Chowdhury, MBBS, FCPS (Medicine), MD (Internal Medicine), Professor \& Chairman, Department of Internal Medicine, Bangabandhu Sheikh Mujib Medical University, Shahbagh, Dhaka 1000, Bangladesh.

Corresponding Author :

Dr. Farzana Shumy, MBBS, FCPS (Medicine)

Medical Officer, Department of Internal Medicine,

Bangabandhu Sheikh Mujib Medical University,

Shahbagh, Dhaka 1000, Bangladesh.

Email: farzanashumy@hotmail.com as well as collagen vascular disorder. Moreover, it is one of the differential diagnoses of pyrexia of unknown origin.

\section{Case Presentation:}

A 14-year-old boy presented for evaluation of fever for three months. He also had anorexia, malaise and fatigue. Apart from these, there were no organ specific symptoms and no significant past and family history. He did not travel recently. Physical examination showed moderate anaemia and raised temperature $\left(102^{\circ} \mathrm{F}\right)$. He had hepatosplenomegaly (3 and 20 $\mathrm{cm}$ respectively), but there was no peripheral lymphadenopathy or ascites. Laboratory data at the time of presentation showed haemoglobin of $8 \mathrm{gm} / \mathrm{dl}$, white cell count 1,800/cumm and platelets 100,000/cumm. Blood film revealed pancytopenia. ESR was only $5 \mathrm{~mm}$ in first hour. He had deranged liver function test (ALT $202 \mathrm{U} / \mathrm{L}$, AST $160 \mathrm{U} / \mathrm{L}$, Alkaline phosphatase (ALP) $220 \mathrm{U} / \mathrm{L}$, lactate dehydrogenase $911 \mathrm{U} / \mathrm{L}$ ) with normal coagulation profile (INR 1.31). Detailed febrile work up was negative for infection like malaria, typhoid and kala-azar. Bone marrow study revealed normal active marrow. Bone marrow trephine biopsy showed only few macrophages.

His chest $\mathrm{x}$-ray was normal and CT scan of abdomen showed huge hepatosplenomegaly and abdominal lymphadenopathy (periportal and prepancreatic). During his hospital stay, his haemogram deteriorated rapidly. At one time, haemoglobin became $6.5 \mathrm{~g} / \mathrm{dl}$, TC $1,500 / \mathrm{cmm}$, platelets $15,000 / \mathrm{cmm}$. It was very difficult to maintain his blood count despite repeated transfusion. He remained febrile with further derangement of liver function test (Total bilirubin $64.4 \mu \mathrm{mol} / \mathrm{L}$, ALP 874 $\mathrm{U} / \mathrm{L}$ ). He remained persistently hyponatremic (Serum $\mathrm{Na}$ varied from 123 to $127 \mathrm{mmol} / \mathrm{L}$ ) since his admission. Fasting triglyceride level found very high $(5.19 \mathrm{mmol} / \mathrm{L})$ as well as serum ferritin $(11022 \mathrm{ng} / \mathrm{ml})$ and plasma fibrinogen was low $(110 \mathrm{mg} / \mathrm{dl})$. Repeat bone marrow trephine biopsy revealed ring granuloma and evidence of haemophagocytosis [Fig.1]. Biopsy materials, sent for mycobacterial, fungal culture and PCR for mycobacterium tuberculosis, were all negative. 


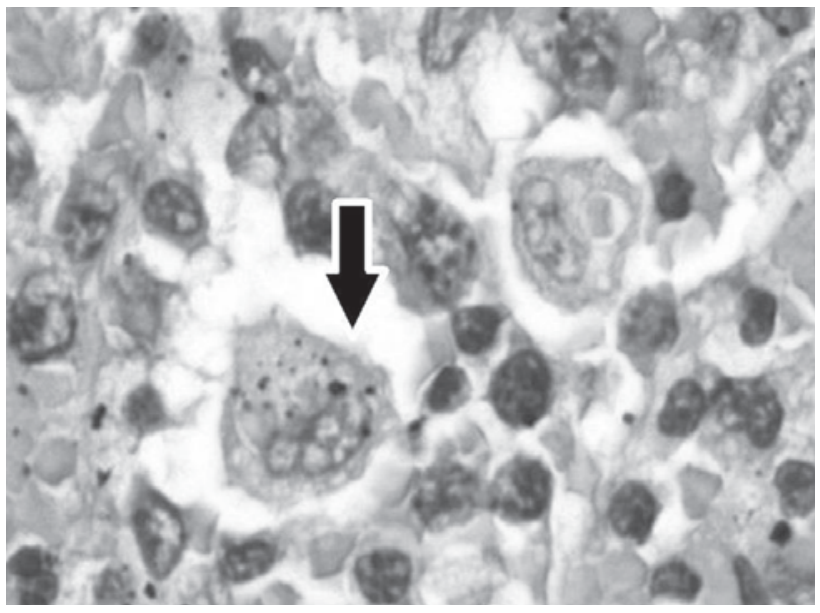

Figure 1 : Bone marrow aspirate (H\&E stain, light microscope, 1000x) showing presence haemophagocyte (black arrow)

Laparoscopic lymph node biopsy demonstrated focal haemophagocytosis with negative immunohistochemistry for lymphoma. Several investigations were done to find out the cause of Haemophagocytosis. ANA, HIV serology, Anti-EBV-IgM, Widal and Weil-Felix tests were negative and complements level were also normal.

There was a plan to start specific chemotherapy. But despite of ongoing treatment with dexamethasone and antibiotics, patient condition rapidly deteriorated and finally succumbed to his disease.

\section{Discussion:}

Haemophagocytic Lymphohystiocytosis (HLH) is a disorder characterised by activation of mononuclear phagocyte system cell, with haemophagocytosis in bone marrow and the rest of the reticuloendothelial cell. ${ }^{1,2}$ The syndrome, has also been referred to as histiocytic medullary reticulosis, was first described in $1939 .^{3}$

This syndrome can be either primary/familial (familial hemophagocytic lymphohistiocytosis- FHL) or reactive/secondary. Familial form is an inherited autosomal recessive disorder. Secondary form is triggered mainly by viral, bacterial, fungal and parasitic infection, as well as collagen-vascular disease and malignancies, particularly T-cell lymphoma. ${ }^{2,4}$ Although, familial hemophagolymphohistiocytosis had been reported in adult, most adult hemophagocytosis are likely to be secondary to an underlying disease. ${ }^{5}$

Diagnostic guidelines, as published by the Histiocytic Society HLH study group, include five of the followings: fever, splenomegaly, bicytopenia, hypertriglyceridemia (>3 $\mathrm{mmol} / \mathrm{L})$, and or hypofibrinogenaemia $(<1.5 \mathrm{~g} / \mathrm{L})$, hemophagocytosis without evidence of malignancy, hyperferritinaemia $(>500 \mu \mathrm{g} / \mathrm{L})$, low or absent natural killer cell cytotoxicity and elevated soluble CD25 levels ( $>2400 \mathrm{IU} /$ $\mathrm{mL}){ }^{1}$ Supportive criteria include neurologic symptoms, cerebrospinal fluid pleocytosis, conjugated hyperbilirubinemia and transaminitis, hypoalbuminemia, hyponatremia, elevated D-dimers and lactate dehydrogenase. ${ }^{5}$

Although the entire pathogenesis is not clear enough, current accepted theory involves an inappropriate immune reaction caused by activated T-cells associated with macrophage activation and inadequate apoptosis of immunogenic cells. This result sustained macrophage activation and tissue infiltration and production of IL-1 and IL-6. The resulting inflammatory reaction cause extensive damage and inflammatory cytokines are responsible for the characteristic disease markers such as cytopenias, coagulopathy and high triglycerides. ${ }^{3}$ Absence of hemophagocytes does not exclude the diagnosis. In initial bone marrow smears, only increased monocytes and monohistiocytic cells may be present. ${ }^{2,3}$ In this case, initial bone marrow aspirate failed to detect any phagocytosis but there was many indirect evidence of this syndrome.

Patients, who meet the diagnostic criteria of hemophagocytosis, should go through cultures of blood and urine and chest radiograph to look for foci of military tuberculosis. Effort should be given for looking evidence of infection like EBV, CMV and parvovirus B19, HIV, HHV-6 and also fungus. Infection screening should be based on travelling history and history of animal exposure, like screening for leishmaniasis, brucellosis, rickettsioses, and malaria. $^{4}$

HLH is usually rapidly fatal with death from muliorgan failure, bleeding and sepsis. It is recommended that treatment be started early when there is high clinical suspicion. ${ }^{1}$

\section{Conclusion:}

In every patient presented with prolong fever, pancytopenia and hepatosplenomegaly after excluding common clinical illness, haemophagocytic lymphohistiocytosis should be considered as differential diagnosis.

\section{References:}

1. Ng D, Ghosh N, Hicks LK. Hemophagocytic Lymphohistiocytosis-Late Diagnosis in an Adult Patient. BMJ Case Rep 2009; published online 17 August 2009, doi: $10.1136 /$ bcr.05.2009.1858.

2. Mayordomo-Colunga J, Rey C, González S, Concha A. Multiorgan Failure due to Hemophagocytic Syndrome: A Case Report. Cases $J$ 2008;1(1):209.

3. Bhattacharyya M, Ghosh MK. Hemophagoctic Lymphohistiocytosis - Recent Concept. J Assoc Physicians India 2008;56:453-457.

4. Fisman DN. Hemophagocytic Syndromes and Infection. Emerg Infect Dis 2000;6(6):601-608.

5. Weitzman S. Approach to hemophagocytic Syndromes. Hematology Am Soc Hematol Educ Program. 2011;2011:178-183. 